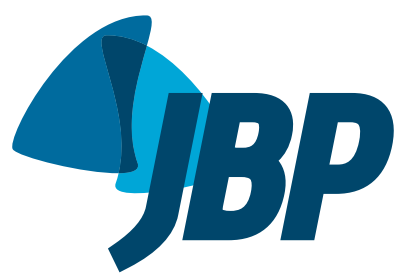

1. Universidade do Estado de Santa Catarina - UDESC - Florianópolis (SC) Brasil.

2. Universidade Federal de Santa Catarina - UFSC - Florianópolis (SC) Brasil.

3. Hospital Sírio-Libanês, São Paulo (SP) Brasil.

4. Hospital Universitário Polydoro Ernani de São Thiago, Universidade Federal de Santa Catarina - UFSC - Florianópolis (SC) Brasil.

a. iD http://orcid.org/0000-0002-8700-202X

b. iD http://orcid.org/0000-0002-6518-6227

c. (iD http://orcid.org/0000-0001-6064-4199

d. (iD http://orcid.org/0000-0002-5390-8739

e. (iD http://orcid.org/0000-0003-4943-2085

f. iD $h t t p: / / o r c i d . o r g / 0000-0002-9811-7172$

g. (iD http://orcid.org/0000-0001-7306-2817

Submitted: 6 June 2016

Accepted: 7 December 2017

Study carried out at the Universidade do Estado de Santa Catarina - UDESC Florianópolis (SC) Brasil.

\section{Validity and reliability of assessing diaphragmatic mobility by area on X-rays of healthy subjects}

\author{
Aline Pedrini1,a, Márcia Aparecida Gonçalves ${ }^{1, b}$, Bruna Estima Leal ${ }^{1, c}$, \\ Michelle Gonçalves de Souza Tavares ${ }^{2, \mathrm{~d}}$, Wellington Pereira Yamagutij ${ }^{3, \mathrm{e}}$, \\ David Luiz Góes ${ }^{4, f}$, Elaine Paulin ${ }^{1,9}$
}

\begin{abstract}
Objective: To investigate the concurrent validity, as well as the intra- and inter-rater reliability, of assessing diaphragmatic mobility by area (DM ${ }_{\text {area }}$ ) on chest $X$-rays of healthy adults. Methods: We evaluated anthropometric parameters, pulmonary function, and diaphragmatic mobility in 43 participants. Two observers (rater A and rater B) determined diaphragmatic mobility at two time points. We used Pearson's correlation coefficient to evaluate the correlation between $\mathrm{DM}_{\text {area }}$ and the assessment of diaphragmatic mobility by distance $\left(\mathrm{DM}_{\text {dist }}\right)$. To evaluate intra- and inter-rater reliability, we used the intraclass correlation coefficient (ICC [2,1]), 95\% Cl, and Bland-Altman analysis. Results: A significant correlation was found between the $D M_{\text {area }}$ and $D M_{\text {dist }}$ methods $(r=0.743 ; p$ $<0.0001)$. For $\mathrm{DM}_{\mathrm{area}^{\prime}}$ the intra-rater reliability was found to be quite high for the right hemidiaphragm (RHD)-ICC $(2,1)=0.92(95 \% \mathrm{Cl}: 0.86-0.95)$ for rater $\mathrm{A}$ and $\mathrm{ICC}(2,1)=$ $0.90(95 \% \mathrm{Cl}: 0.84-0.94)$ for rater $\mathrm{B}$-and the left hemidiaphragm (LHD)-ICC $(2,1)=$ 0.96 (95\% Cl: 0.93-0.97) for rater $A$ and ICC $(2,1)=0.91$ (95\% Cl: 0.81-0.95) for rater $\mathrm{B}$ - $\left(\mathrm{p}<0.0001\right.$ for all). Also for $\mathrm{DM}_{\text {area, }}$ the inter-rater reliability was found to be quite high for the first and second evaluations of the RHD-ICC $(2,1)=0.99(95 \% \mathrm{Cl}$ : 0.98 0.99 ) and ICC $(2,1)=0.95$ (95\% Cl: 0.86-0.97), respectively-and the LHD-ICC $(2,1)$ $=0.99(95 \% \mathrm{Cl}: 0.98-0.99)$ and ICC $(2,1)=0.94(95 \% \mathrm{Cl}: 0.87-0.97)-(\mathrm{p}<0.0001$ for both). The Bland-Altman analysis showed good agreement between the mobility of the RHD and that of the LHD. Conclusions: The DM $M_{\text {area }}$ method proved to be a valid, reliable measure of diaphragmatic mobility.
\end{abstract}

Keywords: Diaphragm/physiology; Validation studies; Reproducibility of results;

Radiography.

\section{INTRODUCTION}

The diaphragm is the main respiratory muscle and is responsible for $70-80 \%$ of ventilation. ${ }^{(1)}$ Therefore, in clinical practice, the evaluation of its mobility is essential to assess the degree of muscle involvement in respiratory and neuromuscular diseases. (2) When a reduction in diaphragmatic motion is identified, it is possible to establish therapeutic strategies to increase muscle movement, as well as to monitor the effectiveness of interventions aimed at improving the functional capacity and quality of life of patients with diaphragmatic dysfunction.

In various clinical settings, it is necessary to evaluate diaphragmatic function in order to diagnose possible malfunctions. Such malfunctions can occur in many situations, including muscular dystrophies, phrenic nerve injury, thoracic surgery, abdominal surgery, and COPD. ${ }^{(3-6)}$ When a reduction in diaphragmatic mobility is identified and objectively measured, it is possible to establish therapeutic strategies to increase muscle movement and to monitor its progression and response to interventions, with the goal of improving functional capacity and quality of life in patients with impaired diaphragmatic mobility. ${ }^{(7)}$

Among the various imaging methods used in evaluating the mobility of the diaphragm, fluoroscopy is considered the most reliable because it provides dynamic images of the diaphragm and direct visualization of diaphragmatic movements in real time. ${ }^{\left({ }^{8}\right)}$ Ultrasound is also considered a valid tool and is widely used in evaluating the mobility of the diaphragm. ${ }^{(9)}$ However, chest X-ray is a noninvasive method that is easily applied and is typically more accessible at hospitals and clinics. Chest $\mathrm{X}$-ray allows direct evaluation of the two hemidiaphragms because it provides static images of the diaphragm. ${ }^{(10)}$

To date, two different methods have been described as means of measuring diaphragmatic mobility on chest X-rays. ${ }^{(11,12)}$ In both methods, two X-ray images are obtained, one at maximum inspiration and the other at maximum expiration, the images then being superimposed and placed on a light box. Although both methods utilize the same images, the measurements are made in different ways. In one of those methods, as

Correspondence to:

Elaine Paulin. Universidade do Estado de Santa Catarina (UDESC), Centro da Ciência da Saúde e do Esporte (CEFID), Departamento de Fisioterapia, Rua Pascoal Simone, 358, CEP 88080-350, Coqueiros, Florianópolis, SC, Brasil.

Tel.: 5548 3664-8602. E-mail: elaine.paulin@udesc.br

Financial support: None. 
described by Toledo et al.,(11) the highest point of one hemidiaphragm at maximum expiration is identified, and a longitudinal line is drawn from that point to the highest point of the same hemidiaphragm at maximum inspiration. The mobility of each hemidiaphragm is then determined by using a caliper to measure the distance between the two points. Hereafter, we refer to that process as the assessment of diaphragmatic mobility by distance $\left(D M_{\text {dist }}\right)$ method. In the other method, as described by Fernandes et al.,(12) after the two images have been superimposed on a light box, tracing paper is placed over the radiographs. The areas represented by the diaphragm in each of the two images are drawn onto the paper and transferred to software that calculates the corresponding area of diaphragmatic mobility. Hereafter, we refer to that process as the assessment of diaphragmatic mobility by area ( $D M_{\text {area }}$ ) method. Although both methods are valid for assessing diaphragmatic mobility, the reliability has been tested only for the $\mathrm{DM}_{\text {dist }}$ as in the study conducted by Toledo et al. ${ }^{(11)}$ and in a previous study conducted by our group. ${ }^{(10)}$ In that study, we evaluated the intra- and inter-rater reliability of the method and found a very high intraclass correlation coefficient (ICC) for all measurements, thus demonstrating that the $D M_{\text {dist }}$ is a reliable method for the evaluation of diaphragmatic motion. When comparing the two methods, it is important to note that the DM ${ }_{\text {area }}$ depends on the use of materials that are simpler and more readily available than are those used in the $D M_{\text {dist }}$. Despite the advantages of the DM ${ }_{\text {area }}$ method, there have as yet been no studies investigating its validity and reliability. Given the importance of and need for every measurement method or instrument to be valid and reliable, together with the lack of studies on the validity and reliability of the $\mathrm{DM}_{\mathrm{area}^{\prime}}{ }^{(12)}$ the objective of the present investigation was to assess the concurrent validity of the $\mathrm{DM}_{\text {area }}$ method to measure diaphragmatic mobility, as well as to assess the intra- and inter-rater reliability of the method in healthy adults.

\section{METHODS}

The present study was approved by the Ethics Committee for Research Involving Human Subjects of Santa Catarina State University (protocol no. 74/2011). All participants gave written informed consent. The study sample consisted of 43 healthy adults between 20 and 59 years of age, who were sampled by convenience, because they lived near or in the catchment area of the hospital where the testing was performed.

To be included in the study, participants needed to have pulmonary function within normal limits. Prospective participants were excluded if they were smokers; were pregnant or suspected of being pregnant; had any cardiorespiratory or neurological disease; had a history of cancer; or were unable to perform any of the study procedures (because of a lack of understanding or cooperation). We also excluded participants for whom the X-ray images obtained did not provide sufficient visibility.
The study was conducted in a physical therapy laboratory and in the radiology department of a public hospital. In the laboratory, participants underwent physical examination, in which anthropometric measurements were recorded, and spirometry was performed on the same day and conducted by a single evaluator. After the initial evaluation, participants were given appointments at the hospital radiology department for the evaluation of diaphragmatic mobility, which was performed by a radiology technician with at least one year of experience.

Participants underwent ventilometry before and during the radiographic examination, to ensure that they performed the respiratory maneuver at the same slow vital capacity (SVC) previously measured. For the DMarea and the DMdist, the mobility of the right hemidiaphragm (RHD) and left hemidiaphragm (LHD) was determined by two observers (rater $A$ and rater B), working independently, at two time points (first and second evaluations, respectively), one-week apart. The same images were analyzed at the first and second evaluations. Both raters were blinded to the identity of the study participants and to the contents of the report provided by the other rater. A third researcher, who was blinded to rater A and B analyses, as well as to which rater was which, made the drawings and used software to perform a random analysis of the area marked by each rater. The X-ray images were analyzed after the completion of all assessments. The RHD was always assessed first.

\section{Anthropometry}

For the measurement of body weight and height, we used a previously calibrated scale (W200/5; Welmy, São Paulo, Brazil) and a stadiometer (Welmy), respectively. The body mass index (BMI) was calculated, from the anthropometric values obtained, as weight in kilograms divided by height in meters squared $\left(\mathrm{kg} / \mathrm{m}^{2}\right)$.

\section{Spirometry}

Spirometry was performed with a portable digital spirometer (EasyOne; ndd Medical Technologies, Zurich, Switzerland), calibrated in accordance with the methods and criteria recommended by the American Thoracic Society. ${ }^{(13)}$ The criteria for normal pulmonary function were an FVC $\geq 80 \%$ of the predicted value, an $\mathrm{FEV}_{1} \geq 80 \%$ of the predicted value, and an $\mathrm{FEV}_{1} /$ FVC ratio $\geq 0.7$.

\section{Ventilometry}

Before and during the radiographic examination, SVC maneuvers were measured with a Wright respirometer (Mark 8; nSpire Health Inc., Hertford, England), with the participants in the supine position. Three SVC maneuvers were performed before the radiographic examination, and the highest value was recorded for later comparison with those obtained during the examination. During the radiographic examination, two SVC maneuvers were performed: from TLC to RV (at the time of the recording of the first X-ray image); and from 
RV to TLC (at the time of the recording of the second $\mathrm{X}$-ray image). The values of the SVC maneuvers were recorded, and the data recorded before and during the radiographic examination were compared in order to determine whether the participants produced the same respiratory effort before and during the evaluation of diaphragmatic mobility.

\section{Diaphragmatic mobility}

In the $\mathrm{DM}_{\text {dist }}$ and $\mathrm{DM}_{\text {area }}$ diaphragmatic mobility was evaluated on the basis of chest X-rays obtained in anteroposterior views. To obtain the X-rays, an experienced, well-qualified radiology technician positioned each participant on an X-ray table in the supine position. Participants were instructed to remain in the same position on the table, moving only their right upper limb to perform the ventilometry maneuver. The radiology technician used a standard distance between the film and the X-ray tube $(1.15 \mathrm{~m})$ for all participants, increasing or decreasing the amount of radiation as required.

Images were recorded on two different films, one obtained at maximum inspiration and one obtained at maximum expiration. Prior to exposure, each film was

Table 1. Anthropometric characteristics and pulmonary function variables of the study participants. ${ }^{a}$

\begin{tabular}{lc}
\multicolumn{1}{c}{ Variable } & (n= 43) \\
Age (years) & $34 \pm 10$ \\
Weight $(\mathrm{kg})$ & $68.77 \pm 15.13$ \\
Height $(\mathrm{cm})$ & $161.45 \pm 36.94$ \\
$\mathrm{BMI}\left(\mathrm{kg} / \mathrm{m}^{2}\right)$ & $24.20 \pm 3.86$ \\
$\mathrm{FVC}$ & \\
$\quad$ & \\
$\quad$ Measured (L) & $5.91 \pm 12.23$ \\
$\quad$ Estimated (\% of predicted) & $94.70 \pm 9.21$ \\
$\mathrm{FEV}_{1}$ & \\
$\quad$ Measured (L) & $3.36 \pm 0.68$ \\
$\quad$ Estimated (\% of predicted) & $93.93 \pm 8.06$ \\
$\mathrm{FEV}_{1} / \mathrm{FVC}$ ratio & \\
$\quad$ Measured & $0.83 \pm 0.07$ \\
$\quad$ Estimated (\% of predicted) & $100.02 \pm 8.15$ \\
\hline
\end{tabular}

BMI: body mass index. ${ }^{a}$ Values are expressed as mean \pm standard deviation. placed into the tray of the X-ray machine, remaining immobile and always in the same position during the exposure. The same researchers accompanied all of the radiographic examinations to their completion, guiding participants in a standardized manner regarding the posture adopted during exposure, providing verbal cues and performing the ventilometry while the images were being obtained. To ensure their safety during the examination, the researchers stood behind a concrete wall with radiological protection barite while guiding the participants through the respiratory maneuvers.

We measured diaphragmatic mobility using the $\mathrm{DM}_{\text {dist }}{ }^{(11)}$ and $\mathrm{DM}_{\text {area }}{ }^{(12)}$ methods. For the $\mathrm{DM}_{\text {dist }}{ }^{\prime}$ after superimposing the images, the rater identified the highest point of one hemidiaphragm (i.e., at maximum expiration) and drew a longitudinal line to the lowest point of the same hemidiaphragm (i.e., at maximum inspiration). The mobility of the hemidiaphragm, as illustrated in Figure 1, was then determined by measuring the distance between the two points with a caliper. ${ }^{(10)}$ The same procedure was followed to measure the mobility of the other hemidiaphragm. To correct for the magnification of the images caused by divergence of the $\mathrm{X}$-rays, the distance between the two points on the radiopaque $X$-ray ruler (in $\mathrm{mm}$ ) was multiplied by 10 and divided by the graduation of the ruler, with the following formula:

$$
\mathrm{CM}(\mathrm{mm})=\frac{\mathrm{MM}(\mathrm{mm}) \times 10(\mathrm{~mm})}{\mathrm{RG}(\mathrm{mm})}
$$

where CM is the corrected mobility, MM is the mobility measurement, and RG is the ruler graduation.

Figure 2 shows the DM ${ }_{\text {area }}$ methodology. After the two images had been superimposed, tracing paper was placed over the X-rays. For each hemidiaphragm, the outlines of the upper, lower, and lateral borders, as well as the right cardiac border in the medial area, were then traced. A 5-cm line was drawn to calibrate the measurement, and the final value was expressed in $\mathrm{cm}^{2}$. The paper images were scanned, and the area corresponding to the displacement of the diaphragm was calculated with the ImageTool for Windows, version 1.28 , developed by the University of Texas Health Science Center at San Antonio. ${ }^{(14)}$
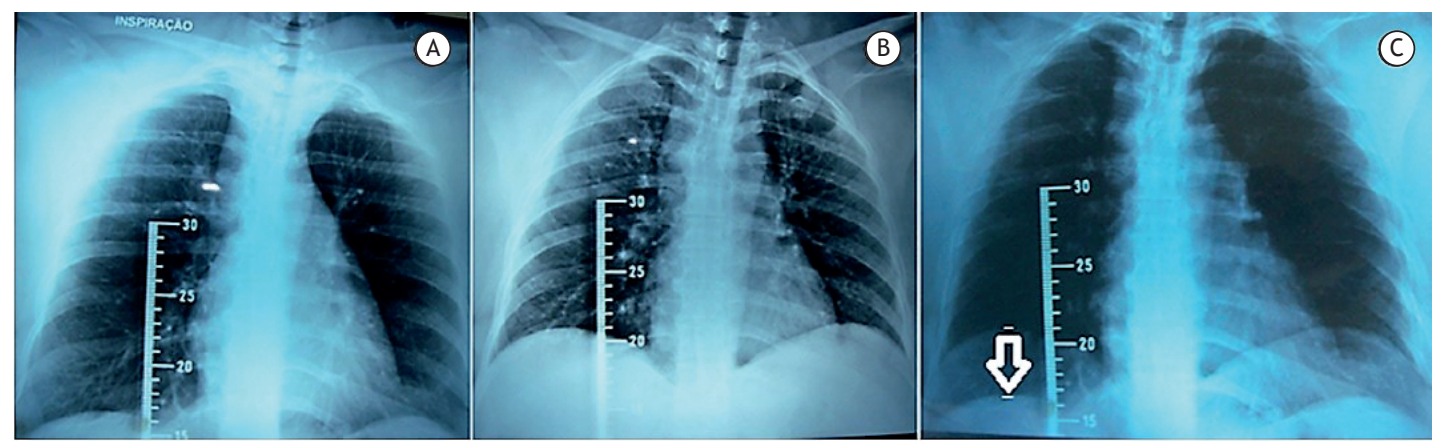

Figure 1. Chest X-rays, in anteroposterior views, used in order to determine the mobility of the right and left hemidiaphragms: A) image obtained at maximum inspiration; B) image obtained at maximum expiration; C) superimposition of images (the image obtained at maximum expiration laid over the image obtained at maximum inspiration), using the radiolopaque ruler as a reference. Source: Saltiel et al. ${ }^{(10)}$ 

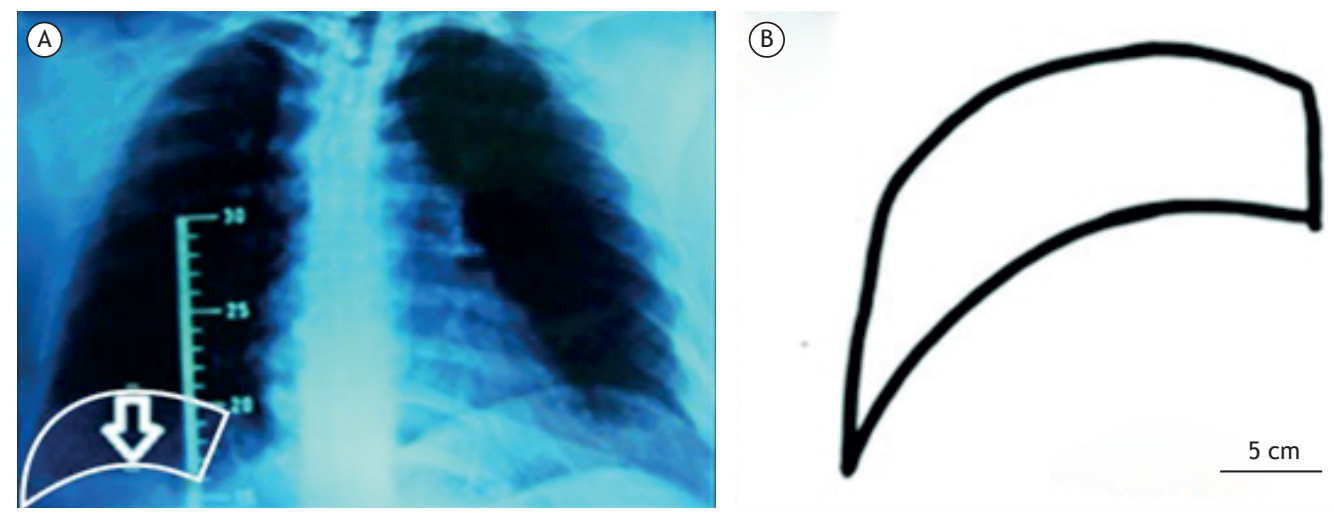

Figure 2. Method of assessing diaphragmatic mobility by area: A) superimposition of images (the image obtained at maximum expiration laid over the image obtained at maximum inspiration); B) Drawing obtained by tracing the left hemidiaphragm at maximum inspiration and at maximum expiration.

\section{Statistical analysis}

The data were analyzed with SPSS Statistics software package for Windows, version 20.0 (IBM Corporation, Armonk, NY, USA). To determine the ideal sample size for this study, a preliminary analysis was carried out, based on the criteria established by Toledo et al. ${ }^{(6)}-r=0.65$; a statistical power of $90 \%$; and $p=$ $0.001-$ which indicated that the appropriate minimum sample size would be 38 subjects. The study included 43 individuals, we are confident that the study had sufficient power to detect statistical significance.

Data were checked for normality with the Shapiro-Wilk test, and the homogeneity of variance was checked with Levene's test. Pearson's correlation coefficient was used in order to quantify the strength of the association between the $\mathrm{DM}_{\text {area }}$ and $\mathrm{DM}_{\text {dist }}$. Paired t-tests were conducted in order to compare the SVC values obtained before and during the radiographic examination. The Wilcoxon test was used in order to compare the mobility of the RHD with that of the LHD. The level of significance was set at 5\% $(p<0.05)$.

For the radiographic measurements, the intra- and inter-rater reliability, based on consistency, was analyzed by determining the two-way 231 mixed-effects ICC (ICC $[2,1])$ values and $95 \%$ confidence intervals (CIs). The ICC $(2,1)$ was interpreted according to the classification system devised by Carter et al. ${ }^{(15)}: 0-0.25$ indicating "little or no reliability"; 0.26-0.49 indicating "low reliability"; 0.50-0.69 indicating "moderate reliability"; 0.70-0.89 indicating "high reliability"; and 0.90-1.00 indicating "very high reliability". Bland-Altman analysis ${ }^{(16)}$ was also conducted to allow better visualization of the agreement between measures.

\section{RESULTS}

A total of 43 participants were evaluated, including 25 females and 18 males. The mean age was $34 \pm 10$ years. Table 1 shows the anthropometric characteristics of the participants and the lung function values obtained.

As can be seen in Figure 3, there was a positive correlation between diaphragmatic mobility determined with the $D M_{\text {area }}$ method and that determined with the $\mathrm{DM}_{\text {dist }}$ method $(r=0.743 ; p<0.0001)$. No statistically significant difference was found between the mean of the SVC maneuvers performed before the radiographic examination and that of those performed during the examination ( $4.36 \pm 0.98$ vs. $4.41 \pm 1.08 ; p=0.17$ ).

In the $D M_{\text {area }}$ method, no statistically significant differences were found between the mobility of the RHD and that of the LHD. For rater A, the DM ${ }_{\text {area }}$ measures of the mobility of the RHD and LHD were $66.12 \pm$ $17.47 \mathrm{~cm}^{2}$ and $67.31 \pm 19.30 \mathrm{~cm}^{2}$, respectively, in the first evaluation ( $p=0.36$ ), whereas they were 64.22 $\pm 15.58 \mathrm{~cm}^{2}$ and $66.66 \pm 18.86 \mathrm{~cm}^{2}$, respectively, in the second evaluation ( $p=0.15)$. For rater $B$, the $\mathrm{DM}_{\text {area, }}$ measures of the mobility of the RHD and LHD were $66.36 \pm 17.44 \mathrm{~cm}^{2}$ and $67.54 \pm 19.34 \mathrm{~cm}^{2}$, respectively, in the first evaluation ( $p=0.37)$, whereas they were $66.93 \pm 16.45 \mathrm{~cm}^{2}$ and $63.88 \pm 17.62 \mathrm{~cm}^{2}$, respectively, in the second evaluation $(p=0.054)$.

The intra-rater analysis indicated that rater $A$ showed "very high reliability" for the evaluation of the mobility of the RHD and LHD-ICC $(2,1)=0.92$ (95\% CI: $0.86-0.90)$ and $\operatorname{ICC}(2,1)=0.96$ (95\% CI: 0.93-0.97), respectively ( $p<0.001$ for both) - as did rater $\mathrm{B}-\mathrm{ICC}$ $(2,1)=0.90$ (95\% CI: $0.84-0.94)$ and $\operatorname{ICC}(2,1)=$ 0.91 (95\% CI: 0.81-0.95), respectively ( $p<0.001$ for both). The inter-rater analysis indicated "very high reliability" between the first and second evaluations of the RHD-ICC $(2,1)=0.99$ (95\% CI: 0.98-0.99) and ICC $(2,1)=0.95$ (95\% CI: 0.86-0.97), respectively ( $p<0.001$ for both)-as well as between the first and second evaluations of the LHD-ICC $(2,1)=0.99$ (95\% CI: 0.98-0.99) and ICC $[2,1]=0.94$ (95\% CI: 0.87-0.97), respectively ( $p<0.001$ for both).

Regarding intra-rater agreement, Figure 4 shows Bland-Altman plots indicating good agreement between the measurements of RHD and LHD mobility obtained by each rater, at the two different time points. These are clinically acceptable values. For the inter-rater agreement, the Bland-Altman plots (Figure 5) indicate good agreement between the measures of RHD and LHD mobility obtained by rater $A$ in the first and second 
radiographic assessments. The measures obtained by rater $\mathrm{B}$ showed good agreement only for the mobility of the RHD.

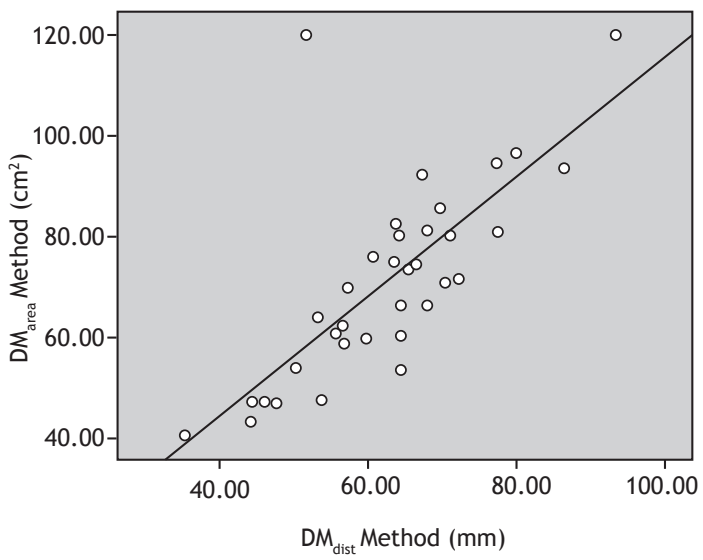

Figure 3. Correlation between diaphragmatic mobility assessed by area (DM area $_{\text {method) }}$ and by distance ( $D M_{\text {dist }}$ method).

\section{DISCUSSION}

In the present study, the $\mathrm{DM}_{\text {area }}$ calculations proved to be valid and reliable, potentially representing an alternative means of assessing diaphragmatic mobility. The DM $M_{\text {area }}$ method allows the two hemidiaphragms to be assessed directly, using static chest X-ray images. It is a simple method that is readily available at hospitals and clinics, which makes it feasible and applicable in research and clinical practice. ${ }^{(10)}$

Although the $\mathrm{DM}_{\text {area }}$ method was not compared with the fluoroscopy method, which is considered the most reliable method, comparisons with the

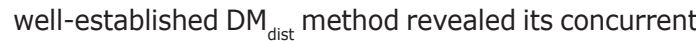
validity in assessing diaphragmatic mobility. ${ }^{(17)}$ We demonstrated a significant correlation between the range of diaphragmatic mobility values obtained with the DM $M_{\text {area }}$ method and that of those obtained with the $D M_{\text {dist }}$ method. We also found the reliability to be very high $($ ICC $[2,1]>0.90)$ for all measurements obtained by both raters. A measure is considered reliable if the ICC is greater than $0.70 .{ }^{(18)}$ Results shown with the

\section{INTRA-RATER AGREEMENT}

\section{RIGHT HEMIDIAPHRAGM}
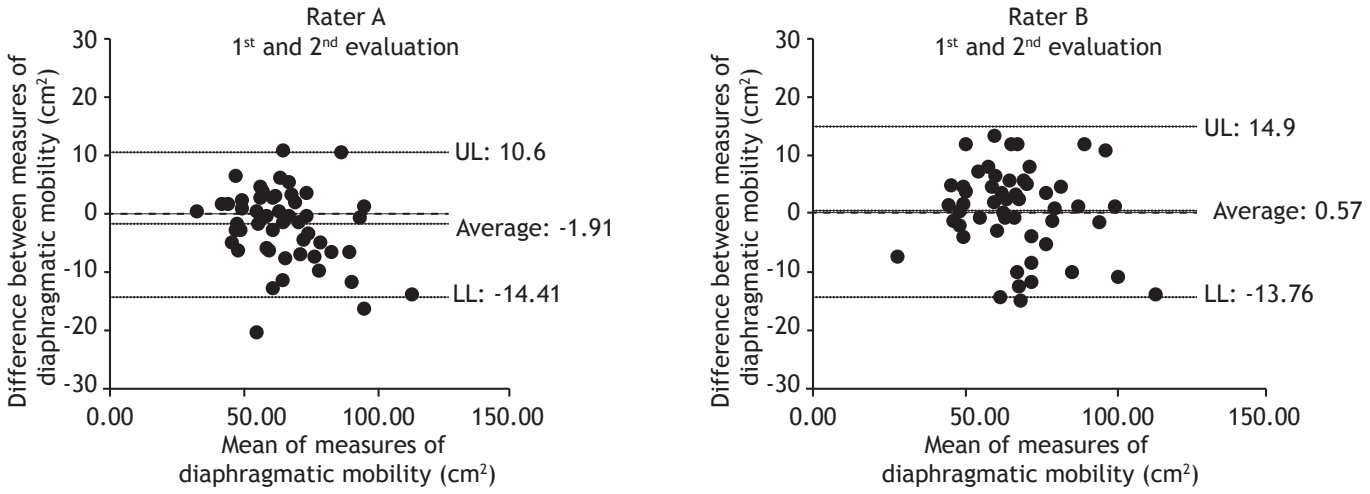

LEFT HEMIDIAPHRAGM

Rater A

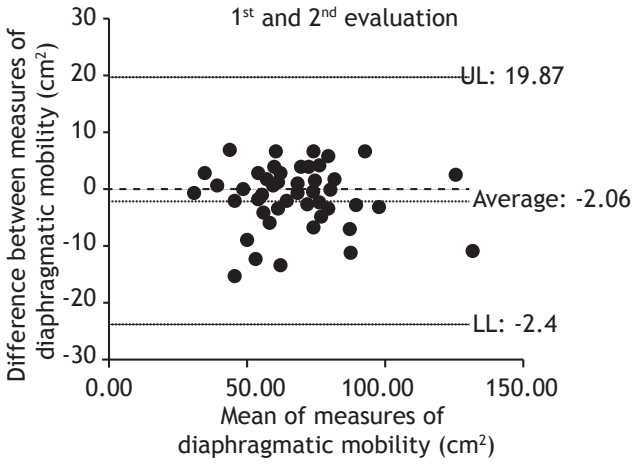

Rater B

$1^{\text {st }}$ and $2^{\text {nd }}$ evaluation

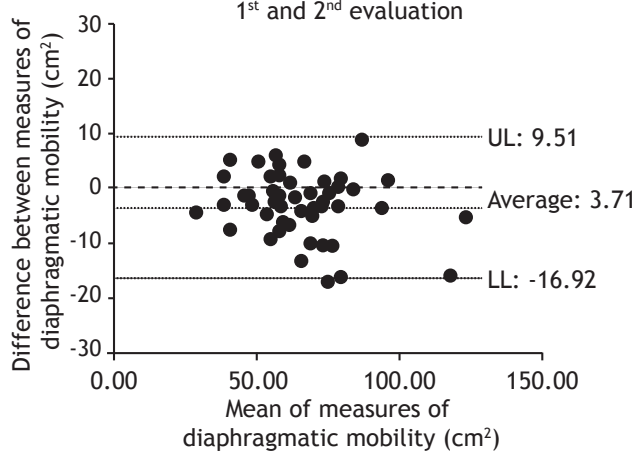

Figure 4. Bland-Altman plots for the analysis of agreement between measures of the mobility of the right and left hemidiaphragms, obtained by rater A and rater $\mathrm{B}$, in the 1 st and 2 nd evaluations (intra-rater agreement). The $\mathrm{x}$-axes show the means of the measures of diaphragmatic mobility (the measure obtained in the 1st evaluation plus the measure obtained in the 2nd evaluation, divided by 2). The y-axes show the differences between the measures of diaphragmatic mobility (the measure obtained in the second evaluation minus the measure obtained in the first evaluation). UL: upper limit; and LL: lower limit. 
INTER-RATER AGREEMENT

RIGHT HEMIDIAPHRAGM
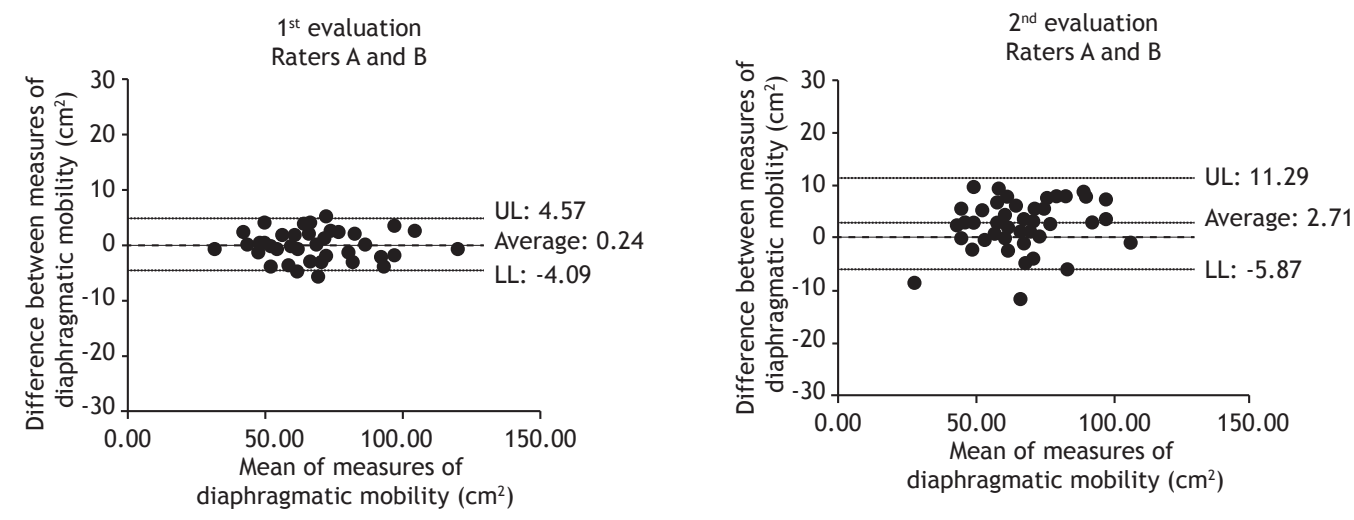

LEFT HEMIDIAPHRAGM
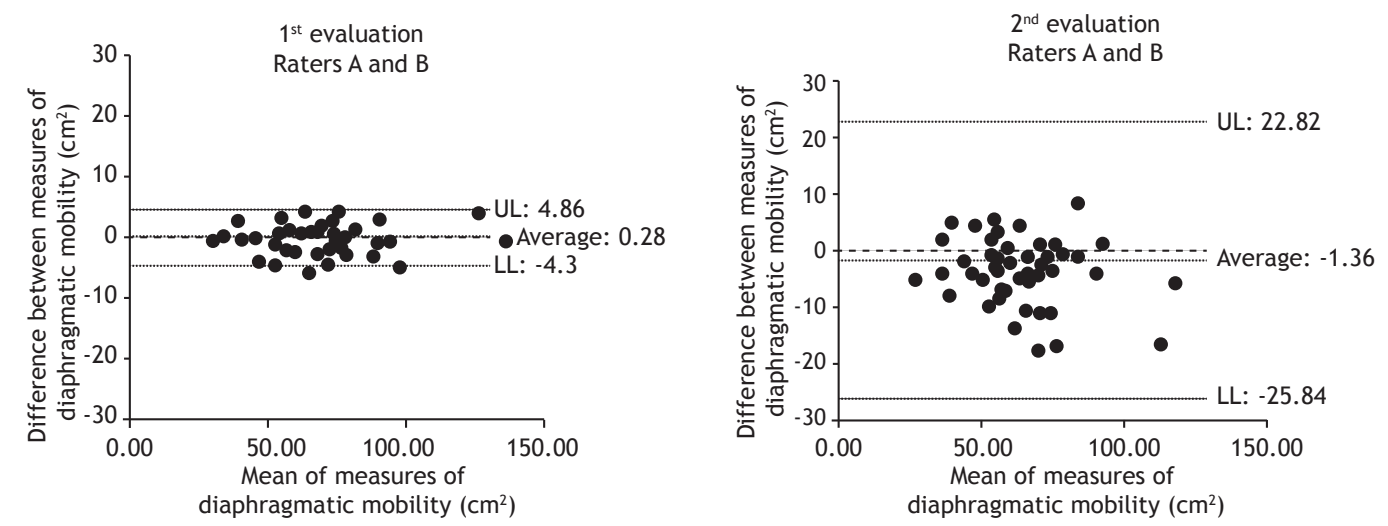

Figure 5. Bland-Altman plots for the analysis of agreement between measures of the mobility of the right and left hemidiaphragms, obtained by raters $A$ and $B$ (inter-rater agreement) in the 1 st and 2 nd evaluations. The $x$-axes show the means of measures of diaphragmatic mobility, obtained by raters $A$ and $B$, for each participant (the measure obtained by rater $A$ plus the measure obtained by rater $B$, divided by 2 ). The $y$-axes show the differences between the measures of diaphragmatic mobility, obtained by raters $A$ and $B$, for each participant (the measure obtained by rater $B$ minus the measure obtained by rater A). UL: upper limit; and LL: lower limit.

Bland-Altman plots support the idea that there was good agreement between the measurements.

An important factor in achieving the high reliability found in our investigation of the DM $\mathrm{area}_{\text {a }}$ method is very likely the methodological rigor adopted by our two evaluators, given that they had been extensively trained in the use of the method, both following the same exact procedures. In addition, the X-ray technician had ample experience conducting radiographic examinations and standardized the technique to be used, always adopting the same distance between the film and the X-ray tube when conducting the examinations.

For inter-rater agreement, the Bland-Altman analysis showed good agreement between the measures of RHD and LHD mobility obtained by rater $A$ in the first and second evaluations. In the analysis of rater $B$, there was good agreement for RHD mobility only. The ICC showed that the inter-rater reliability was very high for the first and second evaluations, confirming the overall good reliability of the DM $\mathrm{Mrea}_{\text {method. }}$
In the present study, there were no significant differences between the mobility of the RHD and that of the LHD. Our results are similar to those reported in other studies. ${ }^{(19,20)}$ In addition, our own research group recently evaluated diaphragmatic mobility by chest X-ray in 42 patients admitted for cholecystectomy and also found no difference in mobility between the two hemidiaphragms of those patients. ${ }^{(10)}$

There was considerable variability between the minimum and maximum values of diaphragmatic mobility obtained in our study (from $40.47 \mathrm{~cm}^{2}$ to 119.94 $\mathrm{cm}^{2}$ ). Other studies have also reported high variability in diaphragmatic mobility, the values ranging from $0 \mathrm{~mm}$ to $97 \mathrm{~mm} \cdot{ }^{(9,19)}$ The possible causes of such variability include the type of sample studied. In the present study, we evaluated not only normal-weight individuals but also those who were obese or overweight, which could have contributed to the wide variability. Obesity is known to hinder respiratory mechanics because a decrease in functional residual capacity due to chest compression results in an elevated diaphragm. (21) 
Therefore, increased mechanical work is required for breathing and the diaphragm acts against the pressure of the distended abdomen, ${ }^{(22)}$ which can limit its mobility. However, it is worth noting that this variability does not affect the applicability of the evaluation method.

The main limitations of this study were the position adopted by the participants for the evaluation of diaphragmatic mobility and the radiographic incidence used. In the study conducted by Fernandes et al., ${ }^{(12)}$ who employed the groundbreaking DM ${ }_{\text {area }}$ method, participants were assessed in the orthostatic position and in a posteroanterior view. In our study, we chose to use the supine position and an anteroposterior view, given that the method most consistently reported in the literature $\left(D M_{\text {dist }}\right)$ evaluates diaphragmatic mobility in that position and view. In addition, more patients are able to assume the supine position than are able to assume the orthostatic position, the use of the former allowing the evaluation of patients in the postoperative period and wheelchair-bound individuals. Although it was not the goal of the study, the results would have been more robust if the method presented had been compared with the gold-standard method (fluoroscopy).

The $D M_{\text {area }}$ method proved to be a valid, reliable tool for assessing the extent of the mobility of the LHD and RHD when compared with the more well-established $D M_{\text {dist }}$ method. This $D M_{\text {area }}$ method is therefore an easyto-use alternative for evaluating diaphragmatic mobility.

\section{REFERENCES}

1. Reid WD, Dechman G. Considerations when testing and training the respiratory muscles. Phys Ther. 1995;75(11):971-82. https://doi. org/10.1093/ptj/75.11.971

2. Yi LC, Nascimento OA, Jardim JR. Reliability of an analysis method for measuring diaphragm excursion by means of direct visualization with videofluoroscopy. Arch Bronconeumol. 2011;47(6):310-4 https://doi.org/10.1016/j.arbr.2010.12.002

3. Maish MS. The diaphragm. Surg Clin N Am. 2010; 90(5):955-68 https://doi.org/10.1016/j.suc.2010.07.005

4. Houston JG, Morris AD, Howie CA, Reid JL, McMillan N. Technical report: quantitative assessment of diaphragmatic movement-a reproducible method using ultrasound. Clin Radiol. 1992;46(6):405-7. https://doi.org/10.1016/S0009-9260(05)80688-9

5. Ayoub J, Cohendy R, Prioux J, Ahmaidi S, Bourgeois JM, Dauzat $M$, et al. Diaphragm movement before and after cholecystectomy: a sonographic study. Anesth Analg. 2001;92(3):755-61. https://doi. org/10.1213/00000539-200103000-00038

6. Paulin E, Yamaguti WP, Chammas MC, Shibao S, Stelmach R, Cukier $A$, et al. Influence of diaphragmatic mobility on exercise tolerance and dyspnea in patients with COPD. Respir Med. 2007;101(10):21138. https://doi.org/10.1016/j.rmed.2007.05.024

7. Yamaguti WP, Claudino RC, Neto AP, Chammas MC, Gomes AC, Salge JM, et al. Diaphragmatic breathing training program improves abdominal motion during natural breathing in patients with chronic obstructive pulmonary disease: a randomized controlled trial. Arch Phys Med Rehabil. 2012;93(4):571-7. https://doi.org/10.1016/j. apmr.2011.11.026

8. Gierada DS, Slone RM, Fleishman MJ. Imaging evaluation of the diaphragm. Chest Surg Clin N Am. 1998;8(2):237-80.

9. Grams ST, Von Saltiél R, Mayer AF, Schivinski Cl, de S Nobre LF Nóbrega IS, et al. Assessment of the reproducibility of the indirect ultrasound method of measuring diaphragm mobility. Clin Physio Funct Imaging. 2014;34(1):18-25. https://doi.org/10.1111/cpf.12058

10. Saltiel RV, Grams ST, Pedrini A, Paulin E. High reliability of measure of diaphragmatic mobility by radiographic method in healthy individuals Braz J Phys Ther. 2013;17(2):128-36. https://doi.org/10.1590/S141335552012005000076

11. Toledo NS, Kodaira SK, Massarollo PC, Pereira OI, Mies S. Right hemidiaphragmatic mobility: assessment with US measurement of craniocaudal displacement of left branches of portal vein. Radiology 2003; 228(2):389-94. https://doi.org/10.1148/radiol.2282011554

12. Fernandes M, Cukier A, Ambrosino N, Leite JJ, Feltrim MI. Respiratory pattern, thoracoabdominal motion and ventilation in chronic airway obstruction. Monaldi Arch Chest Dis. 2007;67(4):209 16.

13. Miller MR, Hankinson J, Brusasco V, Burgos F, Casaburi R, Coates A et al. Standardisation of spirometry. Eur Respir J. 2005;26(2):319-38. https://doi.org/10.1183/09031936.05.00034805

14. UTHSCSA ImageTool for Windows, Version 1.28. San Antonio (TX University of Texas Health Science Center at San Antonio, Image Processing and Analysis Program; 1997.

15. Carter R, Lubinsky J, Domholdt E, editors. Rehabilitation Research: Principles and Applications. 4th ed. Philadelphia: WB Saunders Company; 2013

16. Bland JM, Altman DG. Statistical methods for assessing agreement between two methods of clinical measurement. Lancet. 1986;1(8476):307-10. https://doi.org/10.1016/S0140-6736(86)908378

17. Maher CG, Latimer J, Costa LO. The relevance of cross-cultura adaptation and clinimetrics for physical therapy instruments. Rev Bras Fisioter. 2007;11(4):245-52. https://doi.org/10.1590/S141335552007000400002

18. Freedman D, Pisani R, Purves R. Statistics. 4th ed. New York (NY) WW Norton \& Company; 2008

19. Boussuges A, Gole Y, Blanc P. Diaphragmatic motion studied by m-mode ultrasonography: methods, reproducibility, and norma values. Chest. 2009;135(2):391-400. https://doi.org/10.1378/ chest.08-1541

20. Kantarci F, Mihmanli I, DemireL MK, Harmanci K, Akman C, Aydogan $F$, et al. Normal diaphragmatic motion and the effects of body composition: determination with M-mode sonography. J Ultrasound Med. 2004;23(2):255-60. https://doi.org/10.7863/jum.2004.23.2.255

21. Enzi G, Baggio B, Vianello A. Respiratory disturbances in viscera obesity. Int J Obesity. 1990;14(26).

22. Zerah F, Harf A, Perlemuter L, Lorino H, Lorino AM, Atlan G. Effects the obesity on respiratory resistance. Chest. 1993;103(5):1470-6. https://doi.org/10.1378/chest.103.5.1470 